$\xi=-1$

\title{
An Analytical Model Verification for high Gain H- Slot RPA Using Discontinuities of Radiator
}

\author{
Pritesh Tiwari $^{1 *}$, Lalita Gupta ${ }^{2}$ \\ ${ }^{1}$ Research Scholar, Electronics Department, MANIT, BHOPAL (M.P.) india-462003 \\ ${ }^{2}$ Assistant Professor, Electronics Department, MANIT, BHOPAL (M.P.) india-462003 \\ E-mail:Priteshtiwari.15@gmail.com
}

\begin{abstract}
Antenna plays an important role in most of the RF and microwave applications. Intensifying applications of wireless communications now a day's persist to challenge RF or microwave Antenna with ever more meticulous requirements- smaller size, lighter weight, high performance, economical and easy for fabrication. The advancement in the modern materials fabrication technologies and different research on novel materials, including monolithic microwave integrated circuits (MMIC), low-temperature cofired ceramics (LTCC), hightemperature superconductors (HTS), micro electro mechanic system (MEMS), and micromachining technology, have encouraged the fast strengthening of latest microstrip and supplementary Antenna for RF and microwave applications. The manuscript presented here is a representation of the $\mathrm{H}$ shape patch antenna design and effect of coupling of parasitic patch and discontinuities in radiating structure in order to achieve high gain and better bandwidth $(-10 \mathrm{~dB})$. A mathematical model is presented which has been established on the basis of result obtained. The working frequency range of the manuscript is from $1 \mathrm{GHz}$ to $20 \mathrm{GHz}$.
\end{abstract}

Keywords: Bends Discontinuities (BD), Gaps Discontinuities (GD), Lumped Capacitor, Lumped Inductor, Microelectromechanic system (MEMS), Monolithic Microwave Integrated circuits (MMIC), Open-Ends Discontinuities (OED), Steps Discontinuities (SD).

\section{Introduction}

The theory of microstrip patch antenna was original coined in 1953 by deschamps [1] and a patent for the same is filed in 1955 but the concept gain attention after the advancement in growth of printed circuits, microwave equipment and different kind of low attenuation media material which made microstrip antenna more practical. The microstrip patch antenna Found widespread applications in present days when the compatible devices are widely use in our day to day communication like cell phone, USB dongles, hotspot etc. Thus, physical size reduction and bandwidth enhancement along with high gain are major design consideration $[1,2]$ for practical antenna. In this paper different discontinuities are proposed on an $\mathrm{H}$-shape radiator with the height of $1.6 \mathrm{~mm}$ FR 4 substrate material. On the basis of four designs it has been established a relationship between symmetry of gap discontinuities and high impedance lumped parameter with the gain improvement. It is observed about $27.45 \%$ of gain improvement as compare of the conventional $\mathrm{H}$ slot antenna. The band of radiation is also improved in the proposed design along with satisfactory bandwidth. The working range of frequency is $1 \mathrm{GHz}$ to $20 \mathrm{GHz}$ which cover our most of the day to day communication. The contents of the paper are organized in following manner. Section 2, describe the theory and design equations for strip lines, discontinuities and coupled lines, as well as distributed and lumped components Equivalents for different patch antenna design. Section 3 discuss different $\mathrm{H}$ shape patch antenna with different discontinuities, parasitic patch and patch based lumped parameter which validate the proposed analytical models in section 2 . Section 4 is devoted to the discussion of various results obtained by different proposed design technique. And finally, a conclusion is reached in section 5.

\section{Resonant Characteristics of Microstrip An- tenna}

In common, the design of microstrip antenna associated with the selection of microstrip line and resonating structure, converting transmission line equivalent of lumped element components for an antenna and its characteristics are then obtained by equating the impedances of the transmission lines to the impedances of lumped elements of an earlier designed antenna. The choice of the type of response will depend on the required specifications of antenna. The different parameter which affects the design of microstrip antenna is discussed in this section.

\subsection{Effective Dielectric Constant and Characteristic Imped- ance}

The transmission characteristics of RPA's are well explained by two parameters, first, the effective dielectric constant $\boldsymbol{\varepsilon}_{\text {reff }}$ and second one is the characteristic impedance Zcf, which can be find by quasi-static examination [7] [1],

$$
\begin{aligned}
& \varepsilon_{\text {reff }}=\frac{\varepsilon_{r}+1}{2}+\frac{\varepsilon_{r}-1}{2}\left(1+\frac{10}{p}\right)^{-x y} \\
& \text { where } p=W / h \text {, and } \\
& x=1+0.0204 \ln \left(\frac{p^{4}+(0.0193 p)^{2}}{p^{4}+0.432}\right)+0.0534 \ln \left[1+(0.055 p)^{3}\right]
\end{aligned}
$$




$$
y=\frac{5.64}{10}\left(\frac{\varepsilon_{r}-\frac{9}{10}}{\varepsilon_{r}+3}\right)^{\frac{5.3}{100}}
$$

The accuracy of the model is enhanced by $0.2 \%$ for dielectric constant is less then 128 and width to thickness ration between 0.01 . to 100 i.e $0.01 \leq W / h \leq 100$. The expression for the characteristic impedance is $[7,12]$

$Z_{c f}=\frac{\eta}{6.28 \sqrt{\varepsilon_{e e f}}} \ln \left[\frac{D}{p}+\sqrt{1+\left(\frac{2}{p}\right)^{2}}\right]$

where $p=W / h, \eta=120 \pi \mathrm{ohms}$, and

$D=6+0.28 \mathrm{e}^{\left[-\left(\frac{30.67}{\mathrm{p}}\right)^{0.753}\right]}$

\subsection{Classification of Resonators and Microstrip Discontinuities}

There are different types of resonators, depending upon the shape and performance characteristic of the resonator, which is classified as Single, Dual, Triple and Quadruple mode resonator [6].

Microstrip discontinuities frequently find in the design layout of physical printed antenna which includes steps discontinuities [7,9] open-ends discontinuities, bends discontinuities, gaps discontinuities, and junction's discontinuities. Figure.1, 2, 3 and 4 illustrates all of the above discontinuities design and their equivalent circuits. The property of discontinuities can be repeatedly used [10,11,12] in the patch antenna design.

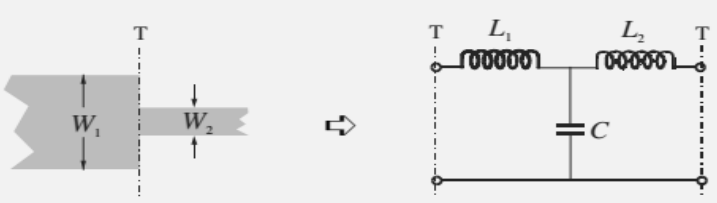

Figure.1 Microstrip step discontinuities.

The capacitance and inductances of the equivalent circuit indicated in Figure $1[6,7]$ for a symmetrical steps can be approximated by the following formulation.

$c=\frac{1.37}{1000} h \frac{\sqrt{\varepsilon_{e e f 1}}}{Z_{c f 1}}\left(1-\frac{W_{2}}{W_{1}}\right)\left(\frac{\varepsilon_{e e f 1}+\frac{3}{10}}{\varepsilon_{e e f 1}-\frac{2.58}{10}}\right)\left(\frac{\frac{W_{1}}{h}+\frac{2.64}{10}}{\frac{W_{1}}{h}+\frac{8}{10}}\right)(p F)$

$L_{1}=\frac{L_{x 1}}{L_{x 1}+L_{W 2}} L, \quad L_{2}=\frac{L_{x 2}}{L_{x 1}+L_{x 2}} L$

Where,

$$
\begin{aligned}
& L_{x i}=Z_{c f i} \sqrt{\varepsilon_{e e f i}} / c \\
& L=\frac{0.987}{1000} h\left(1-\frac{Z_{c f 1}}{Z_{c f 2}} \sqrt{\frac{\varepsilon_{\text {eef } 1}}{\varepsilon_{\text {eef } 2}}}\right)^{2}(\mathrm{nH})
\end{aligned}
$$

Likewise different discontinuity are used for design of microstrip antenna by calculating required parameters.

For an open end microstrip line of width, the different fields present do not stop suddenly however these fields extends vaguely further due to the presence of the fringing field effect. This fringing field effect can be explained either by an equivalent capacitance $\mathrm{Cp}$ connected in shunt or by an equivalent transmission line $[6,7]$ of length $\Delta l$, as shown in Figure 2. The relationship between the two parameters-equivalent can be represented by

$\Delta l=\frac{v Z_{c} Z_{p}}{\sqrt{\varepsilon_{e e f}}}$

Where $v$ is the light velocity in free space. A closed-form expression for $\Delta l / h$ is given by

$$
\frac{\Delta l}{h}=\frac{\varphi_{1} \varphi_{3} \varphi_{5}}{\varphi_{4}}
$$

Where $\varphi_{1}=\frac{43.49}{100} \frac{\varepsilon_{e e f}^{0.81}+0.26(p)^{\frac{85.44}{100}}+\frac{23.6}{10}}{\varepsilon_{e e f}^{0.81}-0.189(p)^{\frac{85.44}{100}}+\frac{87}{10}}$

$$
\begin{aligned}
& \varphi_{2}=1+\frac{(p)^{\frac{3.71}{10}}}{2.35 \varepsilon_{r}+1} \\
& \varphi_{3}=1+\frac{1+\frac{52.74}{100} \tan ^{-1}\left[0.084(w / h)^{1.9413 / \varphi_{2}}\right.}{\varepsilon_{e e f}^{0.9236}} \\
& \varphi_{4}=1+\frac{3.7}{100} \tan ^{-1}\left[\frac{6.7}{100}(p)^{1.456}\right]\left[6-5 e^{\frac{3.6}{10}\left(1-\varepsilon_{r}\right)}\right] \\
& \varphi_{5}=1-\frac{2.18}{10} e^{-7.5 p}
\end{aligned}
$$

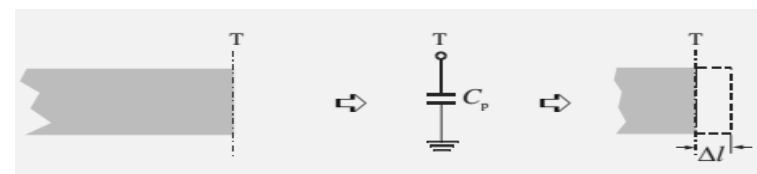

Figure.2 Microstrip open-end discontinuities

The representation of microstrip gap by its equivalent circuit is shown below in Figure 3. Where $C p$ and $C g$ are shunt and series capacitances respectively which can be calculated by

$C p=1 / 2 C r$

$C g=1 / 2 C e-1 / 4 C r$

where

$\frac{C_{e}}{w}\left(\frac{p F}{m}\right)=\left(\frac{10 \varepsilon_{r}}{96}\right)^{\frac{8}{10}}\left(\frac{s}{W}\right)^{m_{0}} \mathrm{e}^{k_{0}}$

$\frac{C_{r}}{w}\left(\frac{p F}{m}\right)=12\left(\frac{\varepsilon_{r}}{9.6}\right)^{\frac{9}{10}}\left(\frac{s}{W}\right)^{m_{e}} \mathrm{e}^{k_{0}}$

$m_{0}=p\left[\frac{6.19}{10} \log (p)-\frac{38.53}{100}\right]$

$k_{0}=\frac{42.6}{10}-\frac{14.53}{10} \log (p)$

$$
\text { for } 0.1 \leq \frac{s}{W} \leq 1.0
$$

$m_{0}=\frac{86.75}{100}$ for $0.1 \leq \frac{s}{W} \leq 0.3$

$k_{e}=\frac{20.43}{10}(p)^{\frac{12}{100}}$

$m_{0}=\frac{1.565}{(p)^{0.16}}-1$ for $0.3 \leq \frac{s}{W} \leq 1.0$

$k_{e}=1.97-\left(\frac{0.03}{p}\right)$

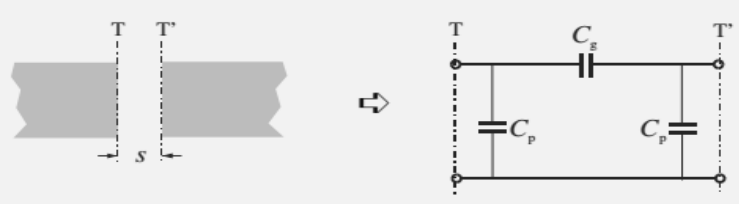

Figure.3 Microstrip gap discontinuities.

The bents of $90^{\circ}$ angle in a microstrips line can be represented by a T-network equivalent, as shown in Figure 4. The closed-form expressions for estimate of capacitance and inductance is given by :

$\frac{C_{e}}{w}\left(\frac{p F}{m}\right)=\left\{\frac{\left(14 \varepsilon_{r}+\frac{125}{10}\right) p-\left(\frac{183}{10} \varepsilon_{r}-\frac{22.5}{10}\right)}{\sqrt{p}}+\frac{2 \varepsilon_{r}}{100 p}\right\}$ for $p<1$

$\frac{C_{e}}{w}\left(\frac{p F}{m}\right)=\left(\frac{95}{10} \varepsilon_{r}+\frac{125}{10}\right) p+\frac{52}{10} \varepsilon_{r}+7$ for $p \geq 1$

$\frac{L}{h}\left(\frac{n H}{m}\right)=400 \sqrt{p}-421$

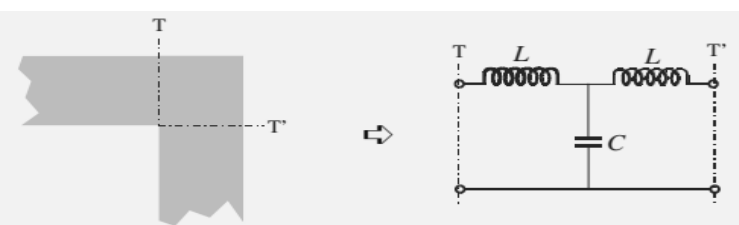

Figure.4 Microstrip bend discontinuities. 


\subsection{Lumped Inductors and Capacitors}

Several emblematic configurations for planar microwave lumped parameters i.e inductors and capacitors $[13,14]$ are shown in Figures 5 and 6.

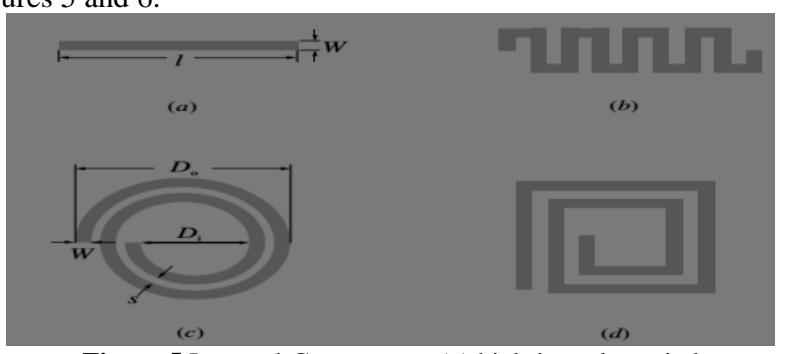

Figure.5 Lumped-Components (a) high-impedance inductor line; (b) meander inductor line; (c) circular spiral inductor; (d) square spiral inductor.

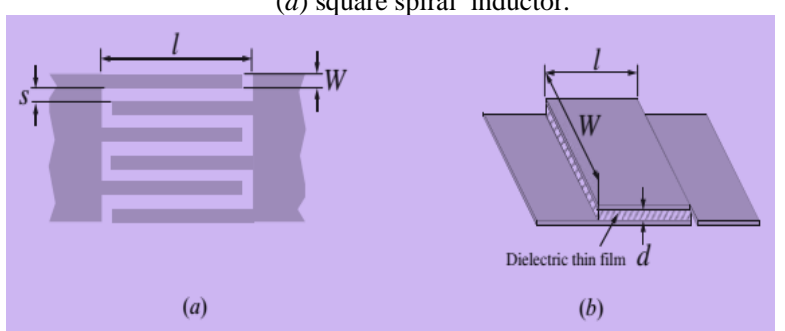

Figure.6 Lumped-Parameter: (a) Inter digital capacitor; (b) MIM capacitor.

\section{Design of the Antenna}

In this section four design of $\mathrm{H}$ slot rectangular patch antenna using FR4 substrate with a dielectric constant of 4.4 is taken. Basic $\mathrm{H}$ slot antenna and then three more designs are investigated by introduction different discontinuities in the radiating patch. Our main aim is to introduce inductive lumped parameter in order to achieve higher Gain and broader bandwidth.

\subsection{Basic H-slot Model}

The basic model of RPA, consist of a very thin metallic strip (patch) which is placed the size of patch is a fraction of a wavelength (usually $0.003 \lambda 0 \leq \mathrm{h} \leq 0.05 \lambda 0$ ) $[1,3]$, above a ground plane, with over all dimension of presented antenna has substrate thickness of $1.6 \mathrm{~mm}$ and size of ground plane and substrate is $32 \mathrm{~mm} \mathrm{X}$ $26 \mathrm{~mm}$. the dimension patch length of $23 \mathrm{~mm}$ and width of $17 \mathrm{~mm}$. an $\mathrm{H}$ shape slot is curved out from $[15,16,18]$ the proposed patch which is shown in the Figure 7.

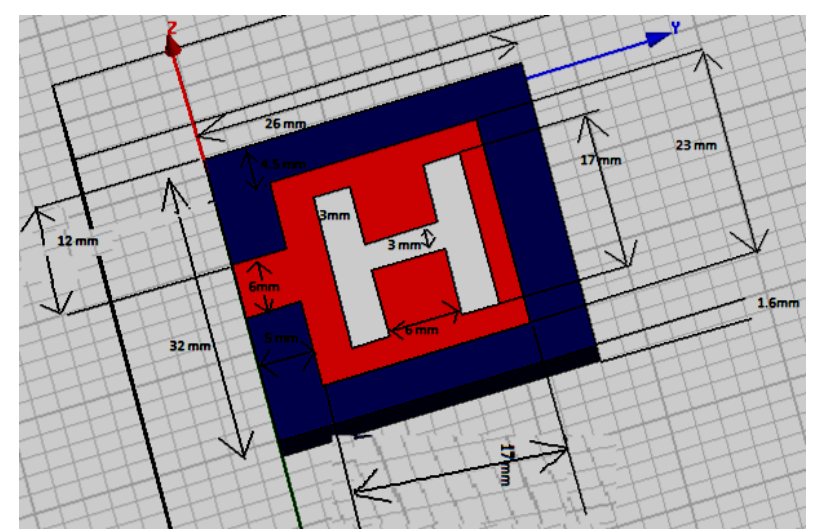

Figure.7 Top view of basic H slot Patch antenna model.

Geometrical parameters of the proposed antenna are optimized through simulations by means of the in ansys-HFSS software. The analysis is based on a frequency range of 2 to $20 \mathrm{GHz}$ which covers most of the communication in our day to day life.

\subsection{H-slot RPA with Step and Gap Discontinuities.}

In this design our basic design is design $\mathrm{A}$ as $\mathrm{H}$ slot along with additional radiating patch of dimension of $23 \mathrm{~mm}$ length and 2 $\mathrm{mm}$ width which act as a high impedance parameter as discuss in section II. The proposed lumped parameter improves the Gain and bandwidth of the proposed antenna. The high $[15,18]$ impedance patch also shift the radiating frequency in accordance with the following relationship $[1,2,3]$.

$f_{c} \cong \frac{1}{2 L \sqrt{\varepsilon_{0} \mu_{0} \varepsilon_{r}}}$

Where $f_{c}$ is the center frequency and other symbol are having usual meaning.

Introduced step discontinuities are introduced in another meander line impedance network are discussed in section II. The detail of the proposed antenna design is shown in figure 8.

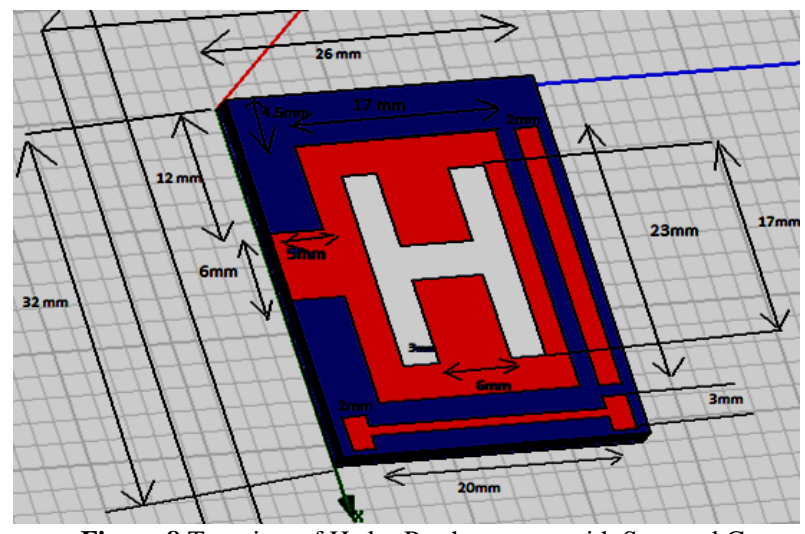

Figure.8 Top view of H slot Patch antenna with Step and Gap Discontinuities model.

\subsection{H -slot RPA with Reduced meander line impedance}

In this design the design parameters of radiating element is almost same as that of the design $B$ the only difference is reduction in meander line impedance which change the gap discontinuities into open end discontinuities and its dimension is symmetrical with two rectangular radiator of dimension $3 \mathrm{~mm}$ X $2 \mathrm{~mm}$ separated by only $3 \mathrm{~mm}$ impedance line as shown in the figure 9 .

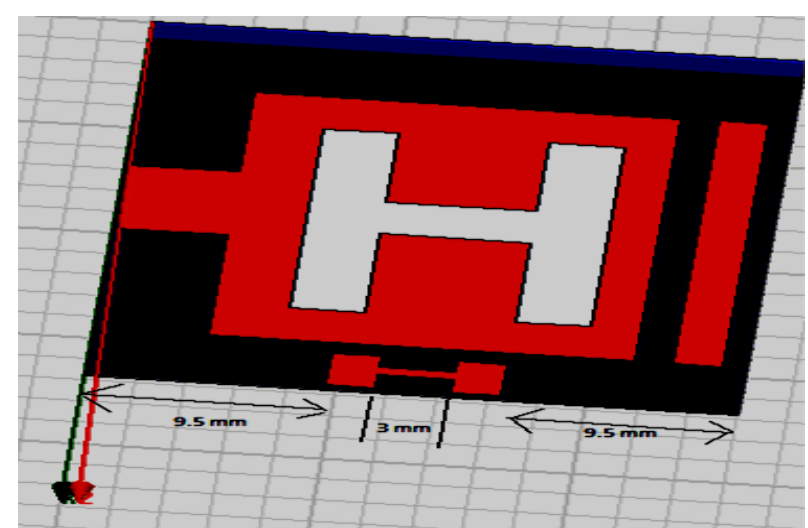

Figure. 9 H slot RPA with Reduced meander line impedance.

\subsection{H -slot RPA with gap symmetry meander line impedance}

In this design the Gap symmetry is maintained with high impedance line of $23 \mathrm{~mm} \times 2 \mathrm{~mm}$ meander line of $13.5 \mathrm{~mm}$ rest of the dimension is same as that of the design $\mathrm{B}$. The proposed design is shown in figure 10 . 


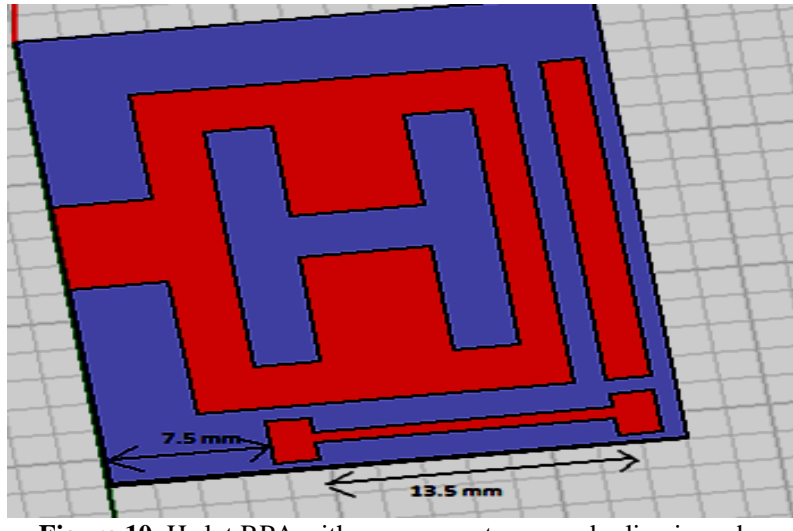

Figure 10. H slot RPA with gap symmetry meander line impedance.

The physical design of proposed antenna is shown in figure 11 .

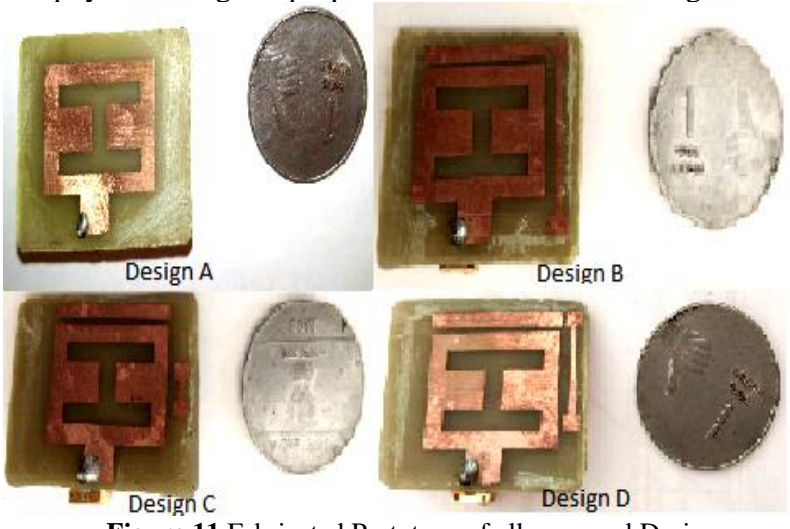

Figure.11 Fabricated Prototype of all proposed Design.

\section{Results and Discussion}

Four performance parameter of antenna (Return Loss, Radiation Pattern, Bandwidth and Gain Peek) for the proposed 4 design are proposed. For design (A) dual band is achieved which shows radiation with center frequency 6.5 and $17.5 \mathrm{GHz}$. On the other hand for design B, C, D Triple band is achieved of radiation the overal results obtained by all four proposed design is shown in table -I.

Table I: Result analysis of Patch antenna

\begin{tabular}{|c|c|c|c|c|c|}
\hline Design & $\begin{array}{c}\text { Radiating } \\
\text { Frequency } \\
(\mathrm{GHz})\end{array}$ & $\begin{array}{c}\text { BW }(- \\
10 \mathrm{~dB}) \\
(\mathrm{MHz})\end{array}$ & $\begin{array}{c}\text { Gain } \\
(\mathrm{dB})\end{array}$ & $\begin{array}{c}\text { Return } \\
\text { Loss }\end{array}$ & VSWR \\
\hline \multirow{2}{*}{$\mathrm{A}$} & 6.5 & 940 & -0.17 & -32 & 1.054 \\
\cline { 2 - 6 } & 17.5 & 2770 & 6.5 & -20 & 1.2 \\
\hline \multirow{3}{*}{ B } & 6.2 & 810 & -0.85 & -29 & 1.074 \\
\cline { 2 - 6 } & 6.8 & 240 & -1.22 & -13 & 1.62 \\
\cline { 2 - 6 } & 17.2 & 2850 & 6.86 & -19 & 1.24 \\
\hline C & 6.2 & 770 & 0.56 & -30 & 1.067 \\
\cline { 2 - 6 } & 6.8 & 220 & 0.5 & -13 & 1.6 \\
\cline { 2 - 6 } & 17.2 & 3010 & 6.56 & -19 & 1.225 \\
\hline D & 6.2 & 800 & -0.83 & -25 & 1.1 \\
\cline { 2 - 6 } & 6.9 & 0200 & 0.75 & -12 & 1.63 \\
\cline { 2 - 6 } & 17.2 & 2840 & 8.96 & -19 & 1.24 \\
\hline
\end{tabular}

The figure 12, 13 and 14 represents the above parameter like return loss S11, -10 dB Bandwidth gain and radiation pattern respectively for design A.

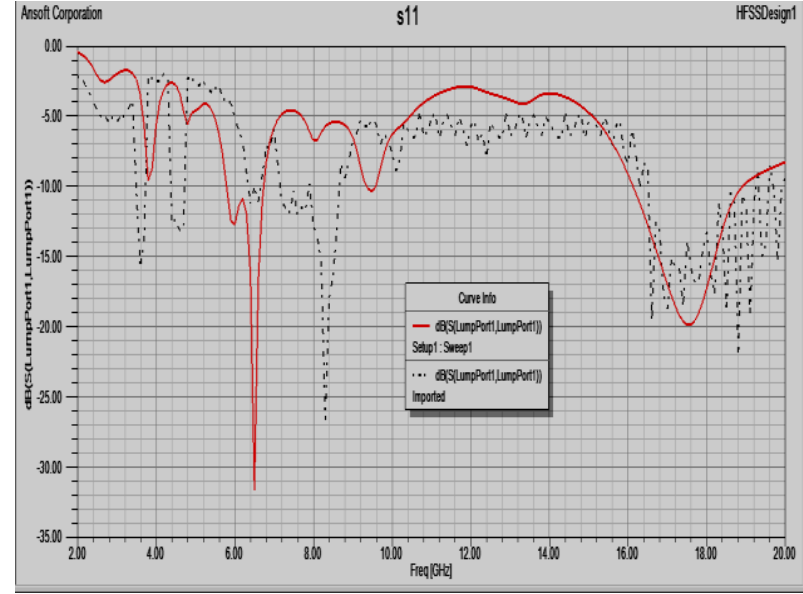

Figure 12. S11 For Design A

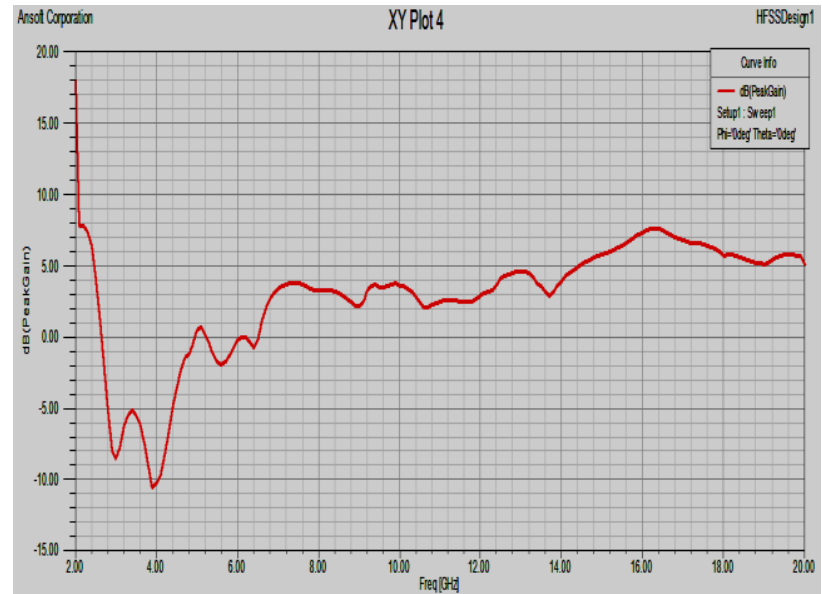

Figure 13. Gain For Design A

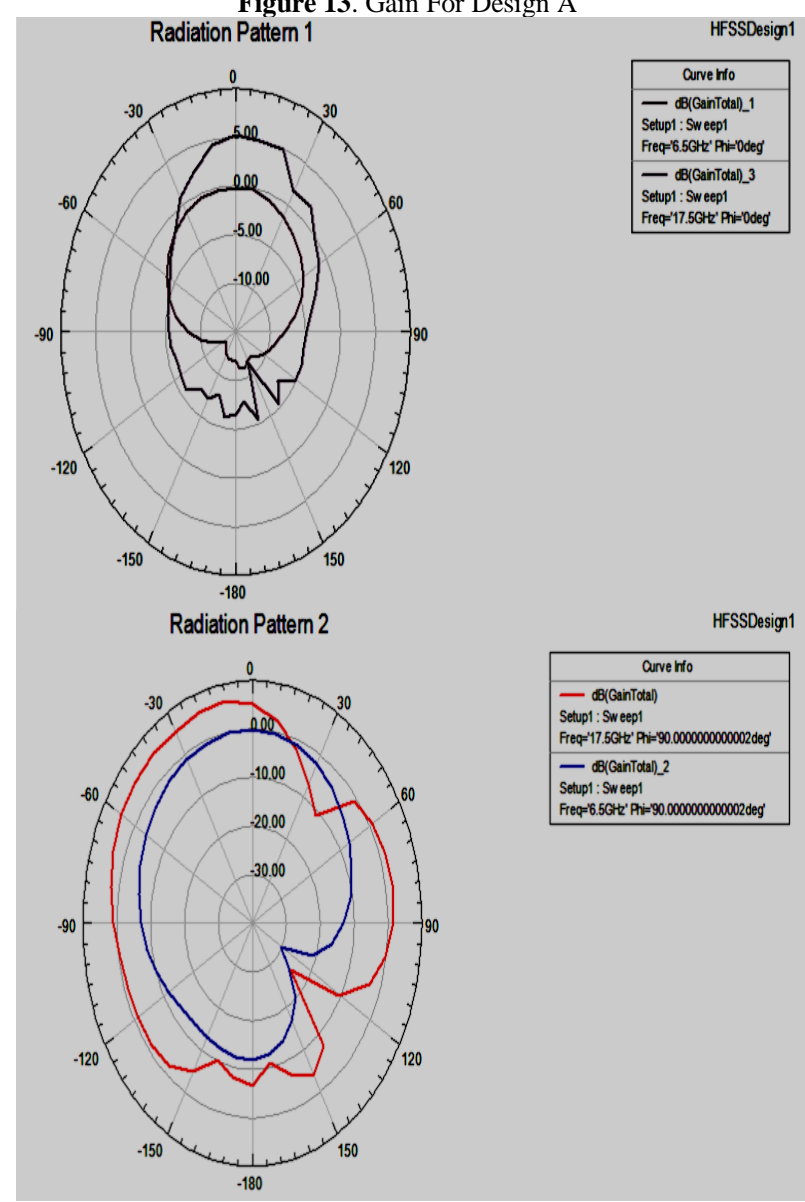

Figure 14. Radiation Pattern For Design A phi $=0^{\circ}$ and phi $=90^{\circ}$ 
The figure 15, 16 and 17 represents the above parameter like return loss S11, -10 dB Bandwidth gain and radiation pattern respectively for design $\mathrm{B}$.

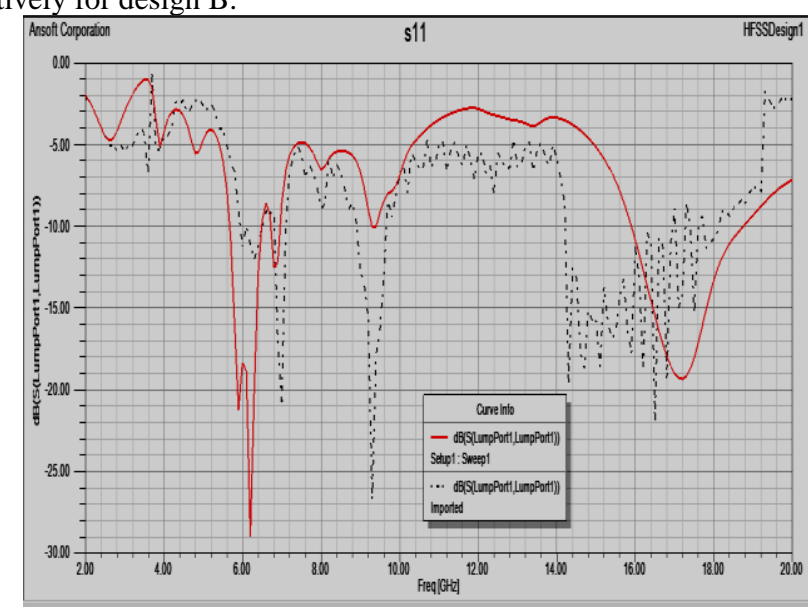

Figure 15. S11 For Design B

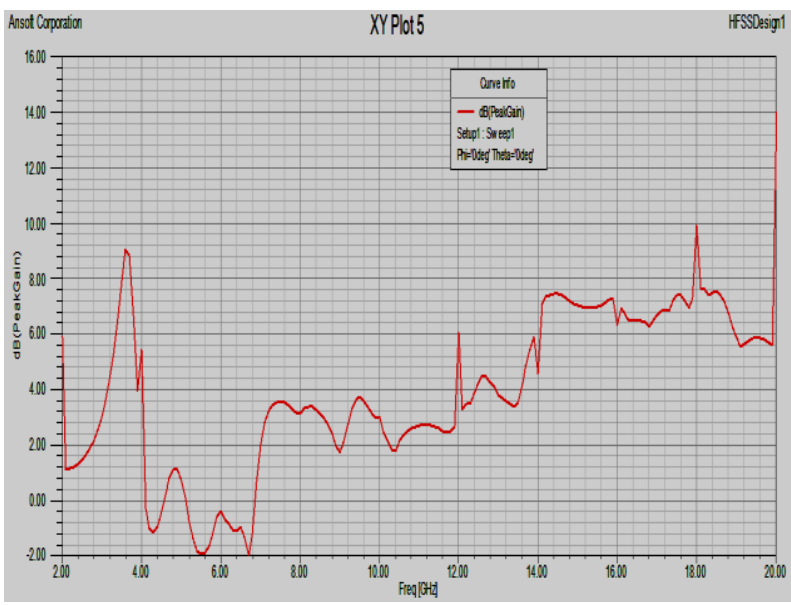

Figure 16. Gain For Design B

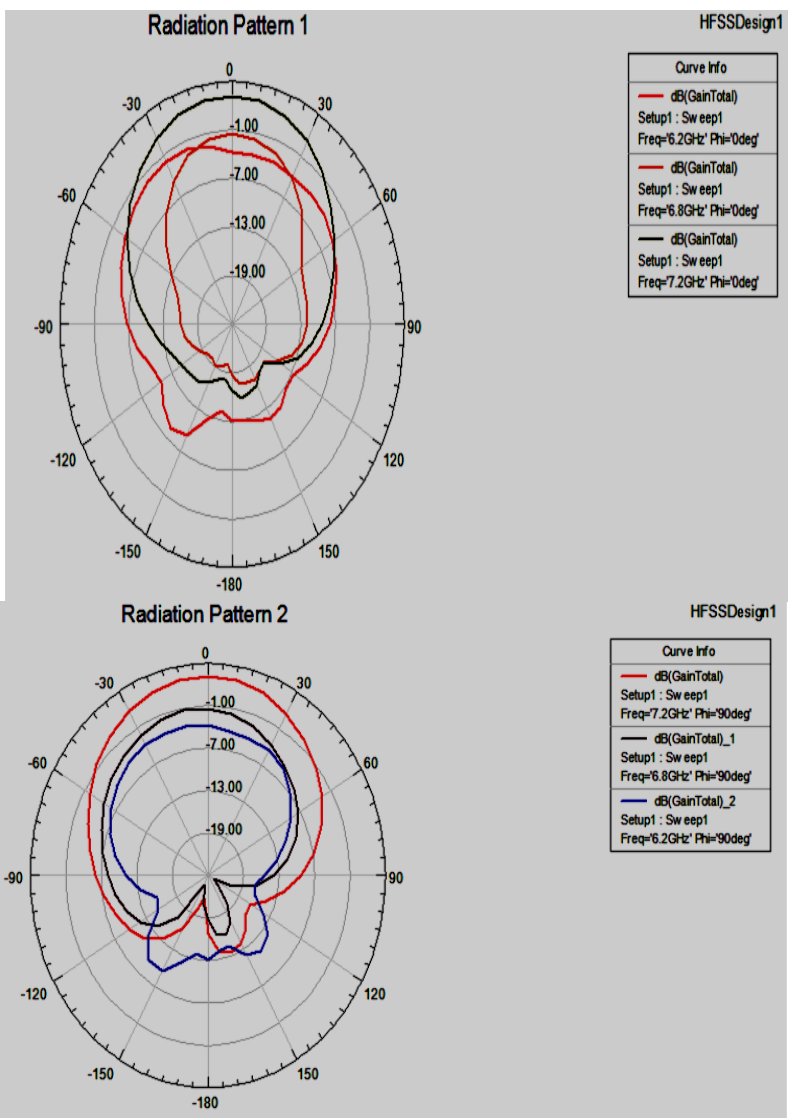

Figure 17. Radiation Pattern for Design $\mathrm{B}$ phi $=0^{\circ}$ and phi $=90^{\circ}$
The figure 18, 19 and 20 represents the above parameter like return loss S11, -10 dB Bandwidth gain and radiation pattern respectively for design $\mathrm{C}$.

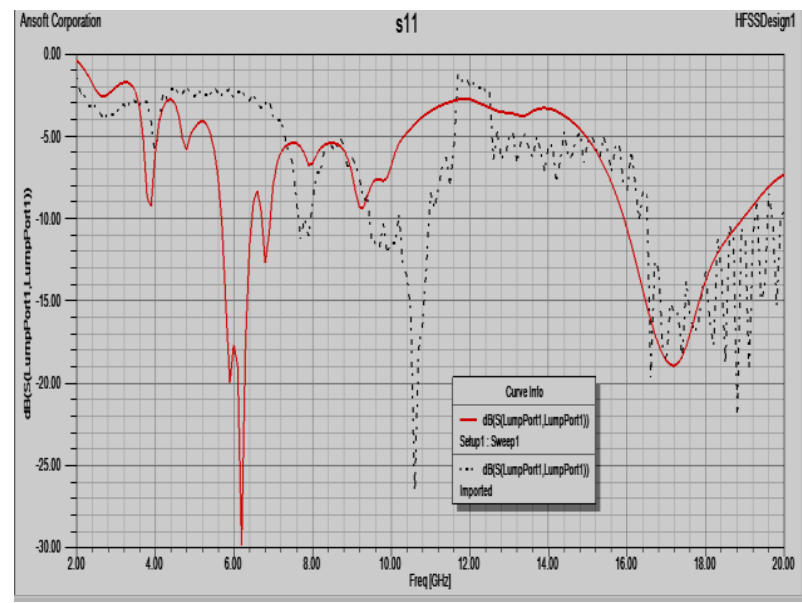

Figure 18. S11 For Design C

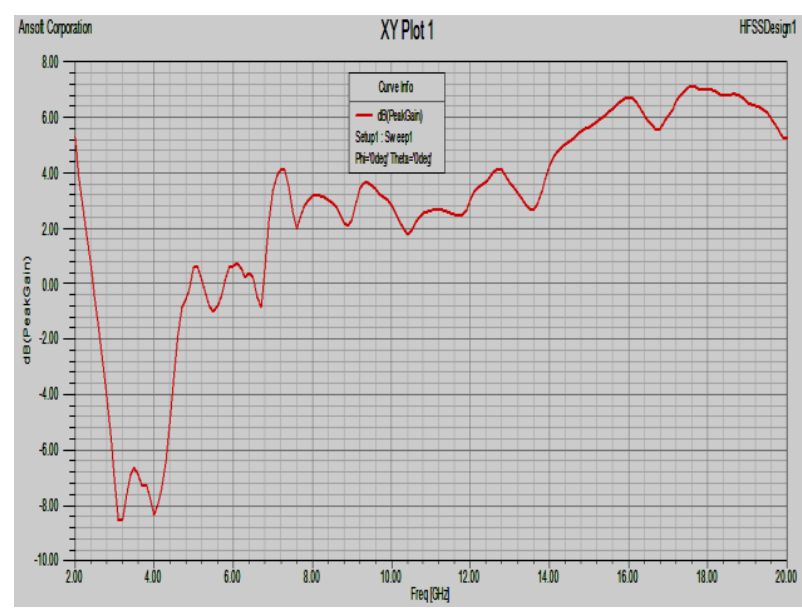

Figure 19. Gain For Design C

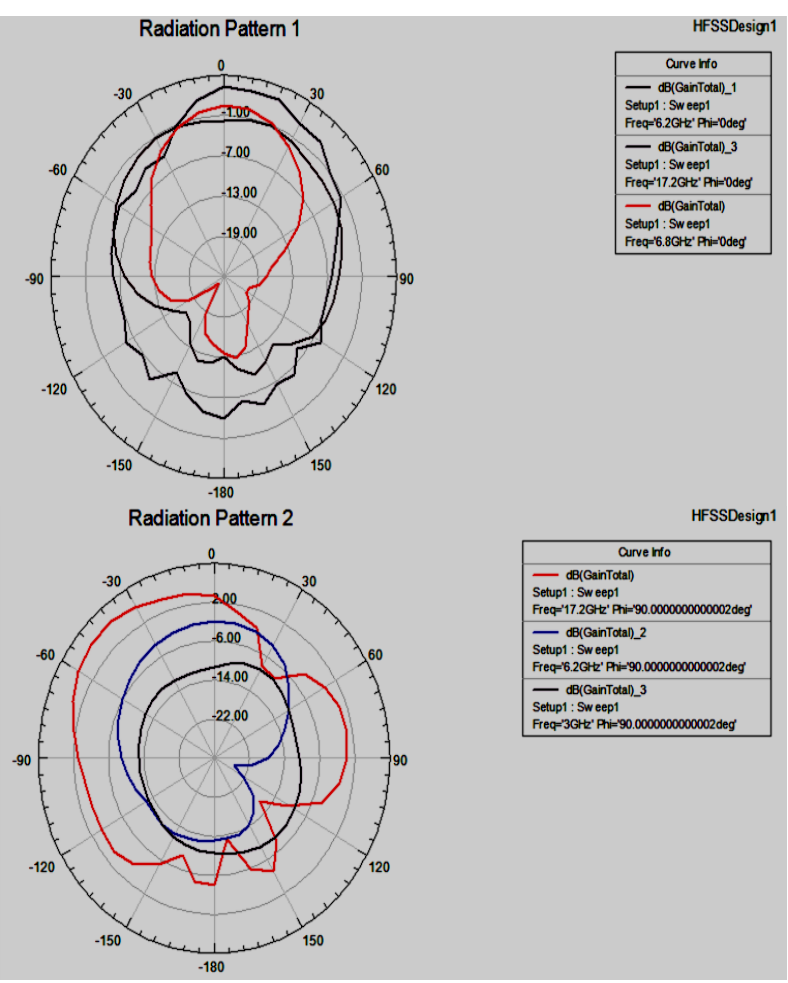

Figure 20. Radiation Pattern For Design $\mathrm{C}$ phi $=0^{\circ}$ and phi $=90^{\circ}$ 
The figure 21, 22 and 23 represents the above parameter like return loss $\mathrm{S} 11,-10 \mathrm{~dB}$ Bandwidth gain and radiation pattern respectively for design D.

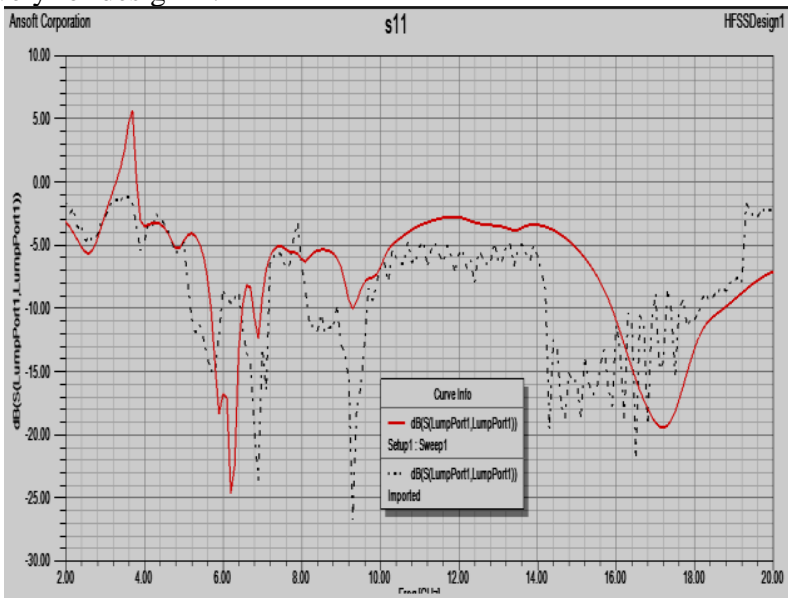

Figure 21. S11 For Design D

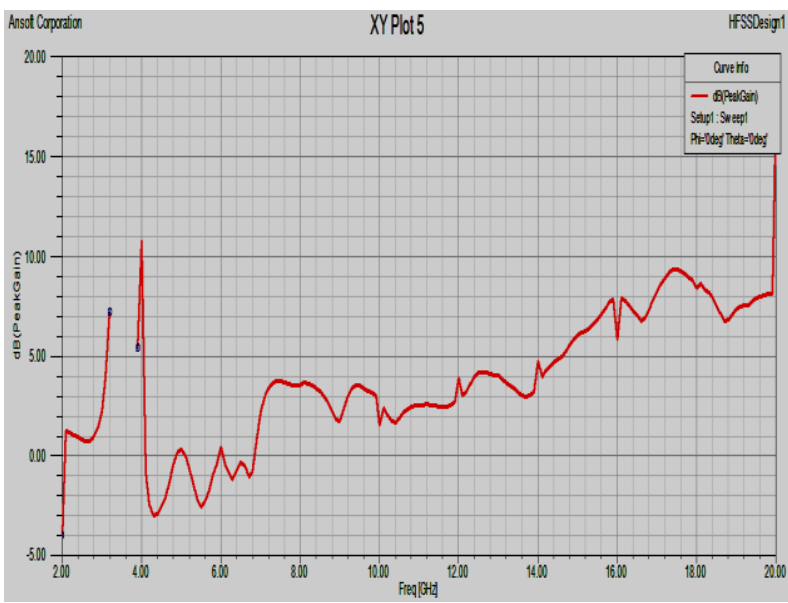

Figure 22. Gain For Design D

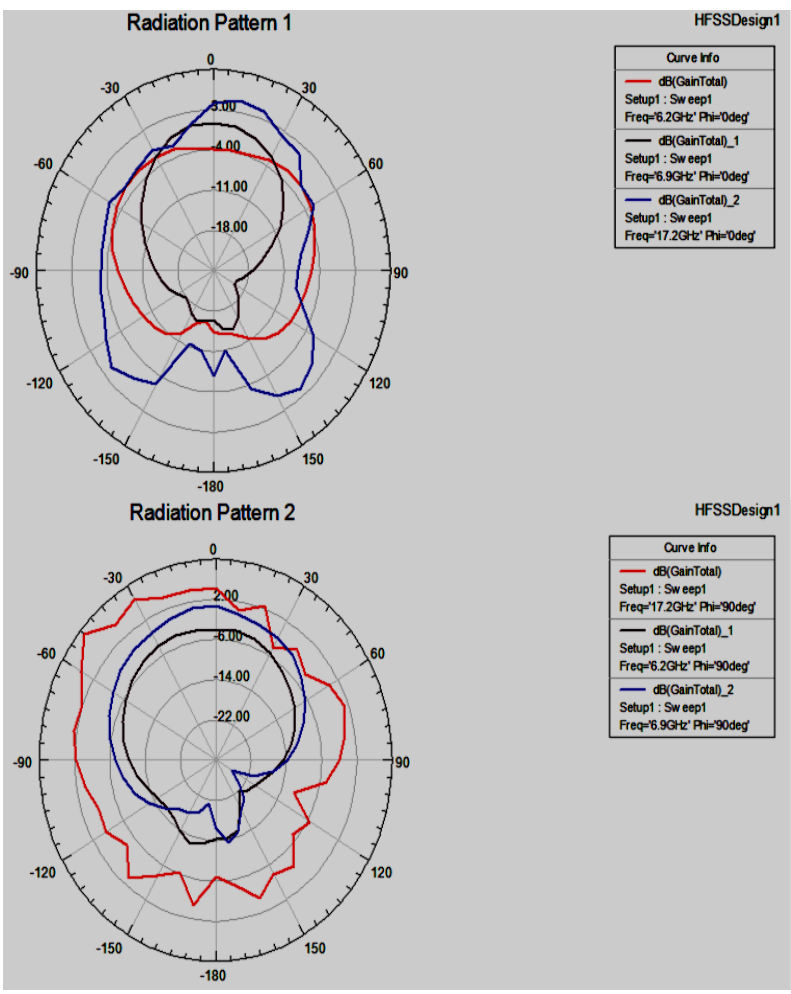

Figure 23. Radiation Pattern for Design $\mathrm{D}$ phi $=0^{\circ}$ and phi $=90^{\circ}$

\section{Conclusion}

A relationship between different discontinuities in the radiation patch has been established by the help of four designs all of them is modeled on a basic H slot RPA. On the basis of obtained results can validate the different analytical model which is presented in section II of this manuscript. After analyzing the results in table-I can conclude that the introduction of different discontinuities and high impedance patch structure increase the Gain with satisfactory bandwidth and return loss. In design $\mathrm{D}$ when the gap is maintained symmetry in discontinuities the highest gain of antenna is about $8.96 \mathrm{~dB}$ with a triple band of radiation with maximum bandwidth of $2840 \mathrm{MHz}$ The gain of antenna starts decreasing as the lumped inductance is reduced as in design $\mathrm{C}$ and it became $6.54 \mathrm{~dB}$ only. In design $\mathrm{B}$ the gain improves to $6.86 \mathrm{~dB}$ just by increasing the meander line impedance. Hence the gap symmetry Lumped parameter patch step discontinuities has a $27.45 \%$ of Gain improvement with triple band radiation than that of conventional $\mathrm{H}$ slot RPA which has only double band of radiation.

In future the research can be extended in field of reduction of mutual coupling between patch antennas by suppressing the surface wave and create different analytical model for the same.

\section{Acknowledgement}

The Authors would like to thank Shri Govindram Seksaria Institute of Technology and Science(SGSITS), Indore for providing the lab instrument(ZVL Network Analyzer $9 \mathrm{KHz}$ to $13.6 \mathrm{GHz}$ ) for result validation.

\section{References}

[1] Constantine A. Balanis; Antenna Theory, Analysis and Design, John Wiley \& Sons Inc. 3rd edition. 2005.

[2] Wong, K. L. "Compact and Broadband Microstrip Antennas". NewYork: J.Wiley and Sons, 2002.

[3] Y.T. Lo. and S.W. Lee, editors, Antenna Handbook Theory, Applications and Design, Van Nostrand Reinhold Company, New York, 1988.

[4] G. I. Kiani, K. L. Ford, and K. P. Esselle et al., "Single-layer bandpass active frequency selective surface," Microw. Opt. Technol. Lett., vol.50, no. 8, pp. 2149-2151, Aug. 2008.

[5] Z. J. Yang, Y. C. Jiao, Z. B. Weng, and L. Zhou, "A compact broadband dual-polarized omnidirectional antenna with high isolations for indoor DAS application," Microwave and Optical Technology Letters, vol. 59, no. 1, pp. 176-180, 2017

[6] K. C. Gupta, R. Garg, I. Bahl, and P. Bhartis, Microstrip Lines and Slotlines, Second Edition, Artech House, Boston, 1996.

[7] T. Edwards, Foundations for Microstrip Circuit Design, Second Edition, Wiley, Chichester, U.K., 1991

[8] C. Deng, Y. Li, Z. Zhang, and Z. Feng, "A wideband highisolated dual-polarized patch antenna using two different balun feedings," IEEE Antennas Wireless Propag. Lett., vol. 13, pp. 1617-1619, 2014.

[9] Weiwen Li, Zhipeng Xia, Baiqiang You, Yanhui Liu, and Qing Huo Liu, "Dual-Polarized H-Shaped Printed Slot Antenna" IEEE Antennas and Wireless Propagation Letters Volume 16, 2017.

[10] Juhua Liu and QuanXue, ’Broadband Long Rectangular Patch Antenna with High Gain and Vertical Polarization" IEEE Trans. on Antennas and Propag., volume 61, no.2, February 2013.

[11] OuYang, J., Yang, F., and Wang, Z.M.: 'Reducing mutual coupling of closely spaced microstrip MIMO antennas for WLAN application', IEEE Antennas Wirel. Propag. Lett., 2011.

[12] Habashi, A., Nourinia, J., and Ghobadi, C.: 'Mutual coupling reduction between very closely spaced patch antennas using lowprofile folded split-ring resonators (FSRRs)', IEEE Antennas Wirel. Propag. Lett., 2011.

[13] Yang, F., and Rahmat-Samii, Y.: 'Microstrip antennas integrated with electromagnetic band-gap (EBG) structures: a low mutual coupling design for array applications', IEEE Trans. Antennas Propag., 2003.

[14] Farahani, H.S., Veysi, M., Kamyab, M., and Tadjalli, A.: 'Mutual coupling reduction in patch antenna arrays using a UC-EBG superstrate', IEEE Antennas Wirel. Propag. Lett., 2010. 
[15] Qi, H., Liu, L., Yin, X., Zhao, H., and Kulesza, W.J.: 'Mutual coupling suppression between two closely spaced microstrip antennas with an asymmetrical coplanar strip wall', IEEE Antennas Wirel. Propag. Lett., 2016.

[16] S. T. Fan, Y. Z. Yin, B. Lee, "Bandwidth Enhancement of a Printed Slot Antenna with a Pair of Parasitic Patches" IEEE Transactions on Antenna and Wireless Propag. vol. 11, 2012.

[17] Shao Wei Liao, QuanXue, Fellow, IEEE, and JianHuaXu"Parallel Plate Transmission Line and L-Plate FeedingDifferentially Driven H-Slot Patch Antenna"IEEE Antennas and Wireless Propagation, vol. 11, 640-644, 2012.

Lalita Gupta is Assistant Professor at the Department of Electronicsand Communication Engineering,Maulana Azad National Institute of Technology, Bhopal .She received her B.E. in Electronics and Telecommunication from Pt.Ravi Shankar Shukla University Raipur,M.Tech in Digital Communication from Maulana Azad National Institute of Technology, Bhopal and $\mathrm{Ph}$.D. from the same institute. Since 2004 She has been associated as a faculty member with the Maulana Azad National Institute of Technology, Bhopal.She is a member of IEEE, IETE, ICEIT,IE. Dr. Gupta has 35 research publications in national and international journals of repute .Her area of Specialization is Signal Processing. 
PriteshTiwari is Assistant Professor at the Department of Electronics and Communication Engineering, University Institute of Technology RGPV, Bhopal .He received B.E. in Electronics and Telecommunication from UIT Barkatullah University ,M.Tech in Digital Communication from Maulana Azad National Institute of Technology, Bhopal and pursuing Ph.D. from the same institute. Since $2002 \mathrm{He}$ has been associated as a faculty member with Different Institutes and have a Teaching Experience around 14 years he is a member of IETE, ICEIT. Mr.Tiwari has 04 research publications in national and international journals of repute .His area of interest is Micro-strip patch Antenna.

E-Mail: Priteshtiwari.15@gmail.com 